\title{
Can Hypo-osmotic Swelling Test (Host) Improve Pregnancy Outcomes in Unexplained linfertility Patients with Normal Semen Parameters Undergoing ICSI -Frozen Embryo Transfer Cycles?
}

\author{
Umit Ozdemir ${ }^{1}$, Zafer Atayurt ${ }^{1} \oplus$, Aytek Sik ${ }^{1}$, Ozkan Ozdamar ${ }^{\circledR}$, Yilda Arzu Aba ${ }^{3}$, Ozan Ozolcay ${ }^{\circledR}$ \\ ${ }^{1}$ Istanbul Sisli Kolan International Hospital, Assisted Reproductive Techniques Center, Istanbul, Turkey \\ ${ }^{2}$ Istanbul Bahcelievler Memorial Hospital, Assisted Reproductive Techniques Center, Istanbul, Turkey \\ ${ }^{3}$ Bandirma Onyedi Eylül University, Faculty of Health Sciences, Balikesir, Turkey \\ ${ }^{4}$ Istanbul Assisted Reproductive Techniques Center, Istanbul, Turkey \\ Correspondence Author: Yilda Arzu ABA \\ E-mail: yildaarzum@hotmail.com
}

Received: 03.02.2021 Accepted: 13.06.2021

\begin{abstract}
Objective: The objective of this study is to compare the pregnancy outcomes of the couples who underwent Hypo-Osmotıc Swelling Test (HOST) as a sperm selection method in Intracytoplasmic sperm injection (ICSI) - Frozen embryo transfer (ET) cycles and those who did not.

Methods: ICSI - Frozen ET cycles, employing HOST as a sperm selection method were assigned to the Study Group; whereas those not employing HOST were included in the Control Group. Both study and control groups were divided into two subgroups according to the age of the women; those between the 25-35 years old and those between 36-40. The study and the control group included 509 and 1304 patients; respectively. Patients between 25-40 years old, who received Invitro fertilization (IVF) treatment for the first time, had good quality (grade A) blastocyst embryo on the 5th day, had normal semen parameters and HOST scores of b, c, and d were included in the study.

Results: Pregnancy outcomes were comparable between cycles using HOST and not using HOST in 25-35 years group. However, cycles employing HOST showed significantly higher pregnancy rate $(p=0.023)$, clinical pregnancy rate $(p=0.005)$, and live birth rate $(p=0.045)$ as compared to cycles not using HOST, in the 36-40 years group.

Conclusion:With normal semen parameters, the use of HOST in ICSI-Frozen ET cycles does not increase live birth rates in women aged 25-35, while it increases the rate of live births in women aged 36-40.
\end{abstract}

Keywords: Hypo-Osmotic Swelling Test, Infertility, Pregnancy Outcomes

\section{INTRODUCTION}

In the area of contemporary assisted reproductive Technologies (ART), the most commonly preferred technique to help infertile couples conceive is the intracytoplasmic sperm injection (ICSI), through which an oocyte is fertilized by a single spermatozoon injection. Although the diagnostic criteria used to define male factor infertility fail to predict under-fertilization in assisted reproductive technology (ART) with excellent accuracy studies, they support the safety and efficacy of ICSI for treating a variety of male factor conditions. (1-3)

The impact of the quality of oocyte and spermatozoon on subsequent fertilization potential and embryo development is undisputed. Sperm selection techniques for Invitro fertilization (IVF) aim to isolate sperm cells that are characterized by a high fertilization potential and can later result in a successful pregnancy. Conventional semen analysis is an essential investigative tool in the evaluation of the male fertility; however the predictive value of this test on reproductive outcomes with ART remains poor (2). Therefore, the need to develop new markers of sperm DNA integrity and the importance of sperm DNA integrity in human fertility is increasingly recognized, and more advanced methods are used to address specific and complex infertility cases to achieve higher live birth rates (4-6).

Intracytoplasmic sperm injection (ICSI) was originally developed as an adjunct to in IVF for couples with severe male infertility, but is now also used in clinical practice in couples with mild male or even unexplained infertility. In the ICSI technique, a spermatozoon is mechanically injected into an oocyte to ensure fertilization. The only sperm selection criteria for ICSI are morphology and mobility as observed under the microscope (7), it has been shown to have significant false-negative rates and sperm cannot predict chromatin integrity (8). In fact, up to $11 \%$ of men with a normal semen analysis have a visible sperm chromatin fragmentation (SCF), which can affect $5 \%$ of men with semen parameters above the 50 th percentile $(9,10)$. For this reason, advanced sperm screening techniques before ICSI attract more attention due to the disappearance of the natural selection process (11). 
Numerous sperm selection methods have been proposed to reduce the possibility of fertilization by DNA-damaged sperm. The hypo-osmotic swelling test (HOST) was originally developed as a diagnostic sperm test to assess the functional integrity of the sperm plasma membrane, as it plays an integral role in sperm capacitation, acrosome reaction, and attachment to the oocyte surface. Water (fluid) passes through the sperm membrane and causes swelling in the tail of living sperm (12) HOST has been proposed as a simple, safe, low-cost and reliable method for determining viable and intact spermatozoa (13) and is currently used as a reliable indicator of sperm quality in clinical practice (1417). Upon spermatozoa's exposure to the hypoosmotic environment, different tail patterns, labeled from " $a$ " to " $g$ " can be observed based on the World Health Organization (WHO) criteria (15).

In this context, HOST has been used in conjunction with ICSI to improve fertilization and pregnancy outcomes in men with severe astheno-zoospermia (18). In ICSI cycles using the HOST method, sperm cells are incubated in a hypo-osmotic solution at $370 \mathrm{C}$. HOST positive spermatozoa (coiled tails) are then selected and washed. When spermatozoa were selected by HOST before ICSI, the absence of total sperm motility, higher fertilization and implantation rates, and better quality embryos were reported, although they were not statistically significant (19-20).

\section{Objectives}

In this study, we aimed to investigate the effect of HOST as a sperm selection method on the reproductive results of couples with normal semen parameters in ICSI - Frozen embryo transfer (ET) cycles.

\section{METHODS}

This retrospective study included 1813 cycles of IVF with ICSI performed at the Istanbul Sisli Kolan International Hospital IVF Unit and Istanbul IVF Center IVF Unit from March 2014 to May 2019. The data of this eligible cycles was obtained by searching for all 24382 IVF cycles that have been performed during this time. Study was approved by the local ethic committee of Bandirma Onyedi Eylul University (08 May, 2020; 2020-23).

\subsection{Patient Selection}

Patients who underwent HOST as a sperm selection method in ICSI-Frozen ET cycles have been included in the study group, whereas patients without HOST in the control group. Both study and control groups were divided into two subgroups according to the age of the women; those between the 2535 years old and those between 36-40. The study and the control groups included 509 and 1304 patients, respectively. Of the 509 patients in the study group, 356 were between the ages of 25-35, and 153 were 36-40. 905 of 1304 patients in the control group were between the ages of 25-35 and 399 of them were $36-40$. Inclusion criteria were as follows; patients between 25-40 years old who underwent their first IVF treatment cycles, couples with normal semen parameters and HOST scores of b, c, and d and the cycles with good quality (grade A) $5^{\text {th }}$ day-blastocyst embryo.

All infertile couples were evaluated to detect any endocrine, chronic inflammatory, infectious, anatomical, and chromosomal abnormalities. Endocrine and metabolic evaluation and tests for diabetes, thyroid diseases, hypopituitarism, hyperprolactinemia, luteal insufficiency, and hyperandrogenism were also made. Transvaginal ultrasound was performed to evaluate pelvic anatomy. Also, rheumatoid factor, C-reactive protein, phospholipids, and cardiolipin (Ig $M$ and $\lg G$ ) antibody levels were evaluated. All patients underwent hysterosalpingography (HSG) for tubal patency or any uterine anomaly evaluation. The karyotypes were studied to detect chromosomal abnormalities. All patients in the study had semen analysis.

Exclusion criteria from the study; patients undergoing fresh embryo transfer, patients with endocrine, chronic inflammatory, infectious, anatomical or chromosomal abnormalities, patients with bilateral or unilateral hydrosalpinx and/or structural anomalies in the uterus, patients who have previously received IVF treatment, those under the age of 25 and above 40 years of age, patients do not have good quality (grade A) blastocyst embryo on the 5 th day, patients who have undergone or need Preimplantation Genetic Screening-Assay / Next Generation Sequencing (PGT-A / NGS), patients who had not received ICSI, patients without HOST score b, $c$ and $d$ and patients with subnormal semen parameters.

\subsection{The HOST Procedure}

As stated in the WHO 2010 criteria, semen samples were prepared and applied for the HOST procedure. 30 minutes after each sample was taken, they incubated at $35{ }^{\circ} \mathrm{C}$ for complete liquefaction. Each sample was evaluated as stated in WHO 2010 criteria, and samples were washed with Multipurpose Handling Medium-Complete (MHM-C) with Gentamicin (Irvine). Then the washed semen sample of each patient was kept at $37{ }^{\circ} \mathrm{C}$ for 5 minutes in 150 mOsm hypoosmotic swelling solutions (Multipurpose Handling Medium diluted with the same amount of purified sterile $\mathrm{H}_{2} \mathrm{O}$ solution). Scoring was performed from "a" to "g" according to the tail shapes formed as a result of tail swellings of sperm. Sperm's response to hypoosmotic stress, scored as grade "a" if lack of tail swelling and grade "b" to "g" according to various degrees of swelling at the distal end of the tail flagellum, was scored (15). In this study, ICSI was applied with sperms that have $b, c$, and d scores.

\subsection{ICSI-ET Procedure}

Controlled ovarian stimulation (COS) was performed with recombinant follicle-stimulating hormone (FSH) (Gonal-F; Merck Serono, Italy) or human menopausal gonadotropin (hMG) (Menopur 75 IU SC/IM, Ferring, USA), with starting doses 
ranging from 225 to $375 \mathrm{IU} /$ day, based on the patients' age and ovarian reserve tests, in a GnRH antagonist protocol. A GnRH antagonist, cetrorelix $0.25 \mathrm{mg}$, (Cetrotide; Merck Serono, Italy) was introduced when a leading follicle achieved $12-13 \mathrm{~mm}$. Final oocyte maturation was induced with 5000 units of IM/SC hCG (Choriomon 5000 IU, IBSA, Switzerland) and/or $0.2 \mathrm{mg}$ of triptorelin (Gonapeptyl daily; Ferring Pharmaceuticals, Saint-Prex, Switzerland) when at least two follicles reached a diameter of 18 $\mathrm{mm}$. Oocyte retrieval was performed $35-36 \mathrm{~h}$ after the trigger.

When the oocyte retrieval was completed, the oocytes were placed into the Continuous Single Culture-NX Complete Medium (CSCM - NXC-IRVINE SCIENTIFIC) and incubated in a Miri Benchtop incubator (ESCO) until $38-40^{\text {th }}$ hour of hCG administration. Than ICSI was performed after this procedure. After the ICSI procedure, all oocytes were transferred into the Continuous Single Culture-NX Complete Medium with 10\% global protein, and their development was followed until the 5 th day. All embryos were cryo-preserved, by vitrification on the 5th day of development, as part of a freeze-all strategy adopted by the IVF Units involved in this study.

Only good quality (Grade A) 5th-day blastocyst embryos were transferred. Embryos were classified as described by the embryo evaluation criteria proposed by ESHRE/ALPHA consensus (21).

Luteal-phase support consisted of $4 \mathrm{mg}$ of oral estradiol valerate (Estrofem tablet, Nova Nordisk, Denmark), $90 \mathrm{mg}$ of vaginal progesterone (Crinone $8 \%$ gel, Merck Serono, England) per day and $50 \mathrm{mg}$ of intramuscular (IM) progesterone (Progestan ampoule, Koçak Farma, Turkey) per day. The estrogen treatment was ceased if a positive pregnancy test was obtained, whereas vaginal and IM progesterone regimen continued until the 12th gestational week.

A positive pregnancy was defined as a serum $\beta$-hCG level $>5 \mathrm{mlU} / \mathrm{ml} 12$ days after embryo transfer. Biochemical pregnancy was described as a serum $\beta$-hCG level of 5-40
$\mathrm{mIU} / \mathrm{ml} 16$ days following embryo transfer. Clinical pregnancy was defined as a visualization of a gestational sac and/or a vital embryo through transvaginal sonography, 4 weeks after embryo transfer. Delivery of a viable infant of 22 weeks or more was considered as live birth, while deliveries below 22 gestational weeks were defined as abortus.

\section{Statistical analysis}

In this study, statistical analysis was performed using the NCSS (Number Cruncher Statistical System) 2007 statistical software (Utah, USA) package program. Descriptive statistical methods, independent t-test in the comparison of two groups with normal distribution, the Mann-Whitney $U$ test in the comparison of two groups without normal distribution, and the Chi-square test in the comparison of categorical variables were used. The results were evaluated as statistically significant if $p<0.05$.

\section{RESULTS}

A total of 509 patients underwent HOST, as a sperm selection method. Among couples who underwent HOST, 356 (19.63\%) were between $25-35$ and 153 (8.43\%) were between 36-40 years of age. A total of 1304 patients were not exposed to HOST; 905 (49.91\%) and 399 (22.00\%) were between 25-35 and between 36-40 of age, respectively. There was no statistically significant difference between the groups in terms of mean ages of women and men ( $p=0.553$ and 0.696 , respectively); Body Mass Index (BMI) ( $p=0.532$ ); infertility duration ( $p=0.254$ ); antral follicle count on the $3^{\text {rd }}$ day of menstruation, Follicular Stimulating Hormone (FSH) and Estradiol $\left(E_{2}\right)$ values $(p=0.446$; 0.600 and 0.128 , respectively); duration of the stimulation ( $p$ $=0.266$ ); number of the retrieved oocytes, mature oocyte and fertilized oocyte counts $(p=0.336 ; 0.372$ and 0.391 ; respectively). Clinical and demographic characteristics of the patients are demonstrated in Table 1.

Table 1. Clinical and Demographic Features of the Patients

\begin{tabular}{|c|c|c|c|c|c|c|}
\hline \multirow[b]{2}{*}{ Clinical features } & \multirow{2}{*}{$\begin{array}{l}\text { Whole group } \\
\qquad n=1813\end{array}$} & \multicolumn{2}{|c|}{$\begin{array}{l}\text { Study Group } \\
\qquad n=509\end{array}$} & \multicolumn{2}{|c|}{$\begin{array}{c}\text { Control Group } \\
n=1304\end{array}$} & \multirow[b]{2}{*}{ P* } \\
\hline & & $\begin{array}{c}25-35 \text { Age } \\
n=356\end{array}$ & $\begin{array}{c}36-40 \text { age } \\
n=153\end{array}$ & $\begin{array}{c}25-35 \text { age } \\
n=905\end{array}$ & $\begin{array}{c}36-40 \text { age } \\
n=399\end{array}$ & \\
\hline Female Age, y & $33.38 \pm 4.47$ & $30.4 \pm 4.51$ & $37.83 \pm 2.51$ & $30.81 \pm 4.25$ & $37.36 \pm 1.56$ & 0.553 \\
\hline Male Age, $y$ & $36.44 \pm 5.52$ & $36.17 \pm 5.69$ & $36.58 \pm 5.23$ & $36.47 \pm 5.81$ & $36.74 \pm 5.37$ & 0.696 \\
\hline BMI & $23.84 \pm 3.99$ & $23.86 \pm 4.37$ & $23.24 \pm 4.15$ & $23.81 \pm 4.12$ & $23.83 \pm 3.97$ & 0.532 \\
\hline Infertility duration, y & $5.35 \pm 3.63$ & $4.91 \pm 3.01$ & $5.83 \pm 4.19$ & $4.11 \pm 3.81$ & $5.30 \pm 4.64$ & 0.254 \\
\hline $\mathrm{D}_{3} \mathrm{AFC}, \mathrm{n}$ & $8.23 \pm 3,45$ & $9.07 \pm 2.41$ & $7.25 \pm 4.28$ & $9.43 \pm 2.73$ & $7.17 \pm 4.39$ & 0.446 \\
\hline $\mathrm{D}_{3} \mathrm{FSH}, \mathrm{mIU} / \mathrm{mL}$ & $8.05 \pm 3.56$ & $8.03 \pm 3.44$ & $8.21 \pm 4.29$ & $8.08 \pm 3.73$ & $8.25 \pm 4.14$ & 0.600 \\
\hline $\mathrm{D}_{3} \mathrm{LH}, \mathrm{mIU} / \mathrm{mL}$ & $8.79 \pm 4.22$ & $9 \pm 4.49$ & $8.54 \pm 3.93$ & $9 \pm 4.26$ & $8.44 \pm 4.01$ & 0.452 \\
\hline $\mathrm{D}_{3} \mathrm{E}_{2}, \mathrm{pg} / \mathrm{mL}$ & $49.29 \pm 27.67$ & $54.37 \pm 32.86$ & $43.62 \pm 19.21$ & $54.58 \pm 32.18$ & $43.03 \pm 19.52$ & 0.128 \\
\hline Stimulation Day, d & $10.74 \pm 2.15$ & $10.64 \pm 1.62$ & $10.86 \pm 2.63$ & $10.23 \pm 1.76$ & $10.13 \pm 2.91$ & 0.266 \\
\hline Number of Oocytes Collected, $n$ & $11.16 \pm 6.78$ & $11.65 \pm 7.23$ & $9.77 \pm 6.09$ & $11.91 \pm 7.12$ & $9.34 \pm 6.61$ & 0.336 \\
\hline Mature Oocytes, $\mathrm{n}$ & $8.83 \pm 5.89$ & $9.91 \pm 6.37$ & $7.88 \pm 5.29$ & $9.89 \pm 6.49$ & $7.76 \pm 5.43$ & 0.372 \\
\hline Fertilized Oocytes, $\mathrm{n}$ & $7.16 \pm 5.21$ & $8.59 \pm 5.99$ & $6.32 \pm 4.35$ & $8.53 \pm 6.14$ & $6.21 \pm 4.12$ & 0.391 \\
\hline
\end{tabular}


Table 2. Pregnancy outcomes of study and control groups by age

\begin{tabular}{|c|c|c|c|c|c|c|c|}
\hline & $\begin{array}{c}\text { Whole group } \\
\qquad n=1813\end{array}$ & $\begin{array}{c}\text { Study Group } \\
25-35 \text { Age } \\
n=356\end{array}$ & $\begin{array}{c}\text { Control Group } \\
\begin{array}{c}25-35 \text { age } \\
n=905\end{array}\end{array}$ & P+ & $\begin{array}{c}\text { Study Group } \\
36-40 \text { age } \\
n=153\end{array}$ & $\begin{array}{c}\text { Control Group } \\
\begin{array}{c}36-40 \text { age } \\
n=399\end{array}\end{array}$ & $\mathrm{P}+$ \\
\hline Pregnancy result, $\mathrm{n}(\%)$ & $1230(67.84)$ & $254(72.19)$ & $637(70.3)$ & 0.210 & $101(66)$ & $238(59.64)$ & 0.023 \\
\hline Biochemical pregnancy, n (\%) & $65(3.58)$ & $13(3.65)$ & $32(3.53)$ & 0.152 & $5(3.26)$ & $15(3.75)$ & 0.414 \\
\hline Ectopic pregnancy, $\mathrm{n}(\%)$ & $15(0.82)$ & $4(1.12)$ & $6(0.66)$ & 0.422 & $1(0.65)$ & $4(1.00)$ & 0.602 \\
\hline Clinical pregnancy, n (\%) & $1150(6.34)$ & $237(66.5)$ & $599(66.18)$ & 0.305 & $95(62.09)$ & $219(54.88)$ & 0.005 \\
\hline Abortion, $\mathrm{n}(\%)$ & $237(13.07)$ & $42(11.79)$ & $111(12.26)$ & 0.208 & $20(13.07)$ & $64(16.04)$ & 0.850 \\
\hline Live birth, $\mathrm{n}(\%)$ & $913(50.36)$ & $195(54.77)$ & $488(53.92)$ & 0.383 & $75(49.01)$ & $155(38.84)$ & 0.045 \\
\hline
\end{tabular}

+: Chi-square Test, $p<0.05$

Of 1813 female patients participating in the study, 1230 (67.84\%) resulted with positive pregnancy tests, 65 (3.58\%) with biochemical pregnancy, 15 (0.82\%) with ectopic pregnancy, 1150 (6.34\%) with clinical pregnancy, 237 (13.07\%) with abortion and 913 (50.36\%) with live birth.

When groups were sub-divided based on age, patients aged 25-35 years with and without HOST the groups were comparable in terms of pregnancy $(p=0.210)$, biochemical pregnancy $(p=0.152)$, ectopic pregnancy $(p=0.422)$, clinical pregnancy $(p=0.305)$, abortion $(p=0.208)$ and live birth ( $p$ $=0.383$ ) rates. Patients aged $36-40$ years with and without HOST, there was no significant difference in the rates of biochemical pregnancy $(p=0.414)$, ectopic pregnancy $(p=$ 0.602 ) and abortion ( $p=0.850)$; however, rates of pregnancy, clinical pregnancy, and live birth were significantly higher in couples having undergone HOST procedure $(p=0.023,0.005$ and 0.045 ; respectively). Reproductive outcomes of the patients are shown in Table 2.

\section{DISCUSSION}

In this study, we showed that the sperm selection by the HOST method in frozen-thawed ICSI cycles increased the rates of positive pregnancy test, clinical pregnancy, and live birth in 36-40 years aged women group, while the rates of ectopic pregnancy and abortion did not alter. However, the results of our study reveal that sperm selection with the HOST method in women aged 25-35 years does not make any difference in the ICSI results, as compared to the control group. The quality of selected sperm for ICSI is thought to be one of the determining factors for the success of assisted reproductive therapy (22). Given the potential of ICSI to overcome severe male infertility, the use of DNA damaged or apoptotic sperms with normal appearance and motility in ICSI may have a negative effect on fertilization and embryo development (23). Therefore, it has been proposed to select sperms not only according to morphology and vitality but also on the basis of functionality, since DNA damaged or apoptotic sperms reduce the chance of fertilizing oocyte $(9,10)$.
Morphological sperm features are most commonly used methods for selecting sperms to fertilize the oocytes in ICSI cycles. In this study, the HOST technique, selecting live immotile sperms, was employed. This technique is not only used for the selection of immotile sperm but also is one of the most common tests to evaluate the structural and functional integrity of the plasma membrane, which is critical for the fertilization process. It evaluates the ability of the sperm plasma membrane to pass water in the hypo-osmotic state, thereby inducing tail swelling and stretching of the plasma membrane (14-24). In the HOST evaluation, the classification varies from " $a$ " to " $g$ " in 7 different ways according to the tail folding of sperm tails $(14,25)$.

It has been stated that Sodium/ Potassium $\left(\mathrm{Na}^{+} / \mathrm{K}^{+}\right)$ andSodium/Hydrogen $\left(\mathrm{Na}^{+} / \mathrm{H}^{+}\right)$pump in the sperm membrane cause tail swelling by reaction under hypotonic conditions. In sperms with intact membranes, the functional activity of these pumps is minimal in tail swelling, and in the sperms with impaired $\mathrm{Na}^{+} / \mathrm{K}^{+}$pump, a complete swelling called HOST grade " $\mathrm{g}$ " is observed $(13,26)$.

Stanger et al. show that it is possible to the selection of sperms with robust DNA based on the degree of the HOST (15). Based on the current results, fertilization of "g" class sperms that contain damaged DNA can be avoided through the HOST rating. This selection may be more useful for severe male infertility cases where the percentage of sperm with damaged DNA is higher $(8,27)$. According to these results, Stanger et al. show that sperm percentage with DNA fragmentation is higher in some HOST degrees (13). For this reason, it is recommended to avoid using " $g$ " grade sperm in ICSI and, where possible, use " $d$ " followed by " $c$ " and " $b$ " grade sperm (28). This is why, in our study, we used the sperms with HOST grade " $b$ ", "c" and " $d$ " in the ICSI procedure. In this study, the HOST method was assessed to evaluate the impact of sperm selection in unexplained infertile couples undergoing frozen-thawed ET-ICSI cycles.

Recently, defects in the functional integrity of the sperm membrane, which can be detected with the HOST test, have been shown to increase the rate of implantation failure or spontaneous miscarriage rather than fertilization failure (29). Moreover, ICSI was proposed as an effective therapy 
for infertility related to subnormal HOST scores, although, poor morphology did not adversely affect the outcome. Remarkably, significant correlations were observed between the percentage of HOST positive sperm and fertilization, implantation, and pregnancy rates (30).

In our study, we compared the results of frozen-thawed ICSI cycles between those who were and were not treated with HOST in a patient population that was categorized by age, unlike the other studies conducted so far. While our study results indicate that there is no significant difference in pregnancy outcomes among patients aged 25-35 with and without the HOST, it also shows a significantly higher pregnancy outcome, clinical pregnancy, live birth rates after the administration of HOST in women between the ages of 36-40. On the other hand, there is no statistically significant difference in biochemical pregnancy, ectopic pregnancy, and abortion rates in both groups.

Unlike other studies, in our study, it was seen that HOST, which is used as a sperm selection method, created more successful pregnancy rates in elderly population (36-40 years old). Sperm DNA damage is seen less, not seen at all or sperm morphology and motility are better in young patients could be the main reason. Based on the results of previous and current studies, sperm selection based on HOST patterns has been shown to improve sperm quality including chromatin integrity, a low level of apoptosis, and sperm morphology (28). The significantly improved implantation and chemical pregnancy rates, and insignificantly improved clinical pregnancy rate were most likely to be due to the quality of sperm selected by the HOST procedure. Of note, previous literature on IVF couples has also observed significant correlations between the percentage of HOST positive sperm with fertilization, implantation and pregnancy rates (30).

We are aware that this study has some limitations. Principally, its retrospective design makes the study prone to the biases inherent to any retrospective data. On the other hand, the inclusion of freeze-all cycles makes it difficult to generalize the results to the fresh-transfer IVF population. Moreover, since the possible contribution of sperm selection to the success of ART has already been established for male-factor infertility, the necessity of a sperm selection technique in an unexplained infertility population, rather than a male-factor population, is a debatable issue.

\section{CONCLUSION}

Based on the results of the present study, we suggest that the use of HOST technique in normo-zoospermic men increases the live birth rate in frozen-thawed ICSI-ET cycles in 36-40 aged women group, but not in 25-35 aged group. It also increases the rates of pregnancy and clinical pregnancy in 36-40 years group. Thus, HOST can be recommended as a sperm selection method in ICSI cycles of unexplained infertile couples, where the female age exceeds 36 . The HOST method in IVF treatments can be considered as a main way to standardized sperm selection procedure for ICSI.

\section{REFERENCES}

[1] Practice Committee of the American Society for Reproductive Medicine, Practice Committee of the Society for Assisted Reproductive Technology. Genetic considerations related to intracytoplasmic sperm injection (ICSI). Fertil Steril 2006;86:103-105.

[2] Guzick DS, Overstreet JW, Factor-Litvak P, Brazil CK, Nakajima ST, Coutifaris C. Sperm morphology, motility, and concentration in fertile and infertile men. N Engl J Med 2001;345:1388-1393.

[3] Tournaye H, Verheyen G, Albano C, Camus M, Van Landuyt $L$, Devroey P. Intracytoplasmic sperm injection versus in vitro fertilization: a randomized controlled trial and a meta-analysis of the literature. Fertil Steril 2002; 78:1030-1037.

[4] Zini A, Jamal W, Cowan L, Al-Hathal N. Is sperm DNA damage associated with IVF embryo quality? A systematic review. J Assist Reprod Genet. 2011; 28(5): 391-397.

[5] Tesarik J, Greco E, Mendoza C. Late, but not early, paternal effect on human embryo development is related to sperm DNA fragmentation. Hum Reprod. 2004; 19(3):611-615.

[6] Barroso G, Valdespin C, Vega E, Kershenovich R, Avila R, Avendaño C. Developmental sperm contributions: fertilization and beyond. Fertil Steril. 2009; 92(3): 835-848.

[7] Nasr-Esfahani MH, Razavi S, Vahdati AA, Fathi F, Tavalaee M. Evaluation of sperm selection procedure based on hyaluronic acid binding ability on ICSI outcome. J Assist Reprod Genet 2008;25:197-203.

[8] Avendan ̃ C, Franchi A, Taylor S, Morshedi M, Bocca S, Oehninger $S$. Fragmentation of DNA in morphologically normal human spermatozoa. Fertil Steril 2009;91:1077-1084.

[9] Zini A, Fischer MA, Sharir S, Shayegan B, Phang D, Jarvi K. Prevalence of abnormal sperm DNA denaturation in fertile and infertile men. Urology. 2002;60(6):1069-1072.

[10] [Belloc S, Hazout A, Zini A, Merviel P, Cabry R, Chahine H, Copin $\mathrm{H}$, Benkhalifa M. How to overcome male infertility after 40: Influence of paternal age on fertility. Maturitas. 2014;78(1):2229.

[11] Said TM, Land JA. Effects of advanced selection methods on sperm quality and ART outcome: a systematic review. Hum Reprod Update 2011;17:719-733.

[12] Mladenovic I, Micic S, Genbacev O. Hypoosmotic swelling test for quality control of sperm prepared for assisted reproduction. Arch Androl 1995;34:163-169.

[13] Stanger JD, Vo L, Yovich JL, Almahbobi G. Hypo-osmotic swelling test identifies individual spermatozoa with minimal DNA fragmentation. Reprod Biomed Online 2010;21:474-484.

[14] Jeyendran RS, Van der Ven HH, Perez-Pelaez M, Crabo BG, Zaneveld LJ. Development of an assay to assess the functional integrity of the human sperm membrane and its relationship to other semen characteristics. J ReprodFertil.1984; 70(1): 219-228.

[15] World Health Organization. Examination and Processing Human Semen, 5th ed. New York: Cambridge University Press; 2010.

[16] Tartagni M, Cicinelli E, Schonauer MM. Males with subnormal hypo-osmotic swelling test scores have lower pregnancy rates than those with normal scores when ovulation induction and timed intercourse is used as a treatment for mild problems with sperm count, motility, or morphology. J Androl 2004;25:781783. 
[17] Ramu S, Jeyendran RS. The hypo-osmotic swelling test for evaluation of sperm membrane integrity. Methods Mol Biol 2013;927:21-25.

[18] Buckett WM. Predictive value of hypo-osmotic swelling test to identify viable non-motile sperm. Asian J Androl 2003;5:209-212.

[19] El-Nour AM, Al Mayman HA, Jaroudi KA, Coskun S. Effects of the hypo-osmotic swelling test on the outcome of intracytoplasmic sperm injection for patients with only nonmotile spermatozoa available for injection: A prospective randomized trial. Fertility and Sterility, 2001; 75(3): 480-484.

[20] Sallam HN, Farrag A, Agameya AF, El-Garem Y, Ezzeldin F. The use of the modified hypo-osmotic swelling test for the selection of immotile testicular spermatozoa in patients treated with ICSI: A randomized controlled study. Human Reproduction, 2005; 20(12): 3435-3440.

[21] Alpha Scientists in Reproductive Medicine, ESHRE Special Interest Group Embryology. Istanbul consensus workshop on embryo assessment: proceedings of an expert meeting. Reprod Biomed Online 2011; 22:632-646.

[22] Henkel R, Schill WB. Sperm preparation for ART. Reprod Biol Endocrinol 2003;1:108.

[23] Tavalaee M, Razavi S, Nasr-Esfahani MH. Influence of sperm chromatin anomalies on assisted reproductive technology outcome. Fertil Steril 2009;91:1119-1126.

[24] Cincik M, Ergur AR, Tutuncu L, Muhcu M, Kilic M, Balaban B. Combination of hypoosmotic swelling/eosin $Y$ test for sperm membrane integrity evaluation: correlations with other sperm parameters to predict ICSI cycles. Arch Androl. 2007; 53(1): 2528.

[25] Rossato M, Galeazzi C, Ferigo M, Foresta C. Antisperm antibodies modify plasma membrane functional integrity and inhibit osmosensitive calcium influx in human sperm. Hum Reprod. 2004; 19(8): 1816-1820.

[26] Peris S, Solanes D, Pen a A, Enric-Rodríguez-Gil J, Riga T. Ionmediated resistance to osmotic changes of ram spermatozoa: the role of amiloride and ouabain. Theriogenology 2000;54:1453-1467.

[27] Avendan ̃ C, Oehninger S. DNA fragmentation in morphologically normal spermatozoa: how much should we be concerned in the ICSI era? J Androl 2011;32:356-363.

[28] Bassiri F, Tavalaee M, Shiravi AH, Mansouri S, Nasr-Esfahani $\mathrm{MH}$. Is there an association between HOST grades and sperm quality? Human Reproduction 2012; 27(8): 2277-2284.

[29] Bhattacharya SM. Hypo-osmotic swelling test and unexplained repeat early pregnancy loss. J Obstet Gynaecol Res 2010;36:119-122.

[30] Check M, Check JH, Summers-Chase D, Swenson K, Yuan $W$. An evaluation of the efficacy of in vitro fertilization with intracytoplasmic sperm injection for sperm with low hypoosmotic swelling test scores and poor morphology. J Assist Reprod Genet. 2003; 20(5): 182-185. 